\title{
471829 - SUCCESFUL MANAGEMENT OF LIFE-THREATENING HEMORRHAGE IN A PATIENT WITH OSTEOGENESIS IMPERFECTA BY LOW-DOSE ACTIVATED RECOMBINANT FACTOR VII
}

\author{
Annick Becotte, $\mathbf{r}^{2}$, P. Moliner, anesthesiologist ${ }^{2}$, G. Devroede, General surgeon ${ }^{3}$, \\ H. Knecht, Hematologist ${ }^{1}$ \\ 1. Anesthesiology, University of Sherbrooke, Sherbrooke, QC, Canada \\ 2. Hematology, University of Sherbrooke, Sherbrooke, QC, Canada \\ 3. General Surgery, University of Sherbrooke, Sherbrooke, QC, Canada
}

Introduction: Osteogenesis imperfecta (OI) is a collagen disorder associated with osseous, dental and tissue brittleness and abnormal bleeding in spite of normal coagulation profile(1). This is the first report of an OI patient with severe intra-abdominal postoperative bleeding successfully treated with low-dose rFVIIa.

Methods: We report the case of a 43-year-old man (agreement obtained) who presented for elective colectomy. The patient had type 1 OI associated with left-sided deafness and caracteristic blue sclerae. He had no history of bleeding diathesis or thrombosis.

Results: The intra-operative hemorrhage was initially thought to arise from a laceration on the spleen and a splenectomy was performed. The ensuing exploration allowed noticing massive diffuse bleeding. The mechanically ventilated patient was transferred to the ICU. Important blood losses persisted through abdominal drains, the absence of blood clotting was observed. Massive transfusions and DDAVP $20 \mu \mathrm{g}$ IV were given without improvement. Confronted with continuing massive haemorrhage, rFVIIa (Niastase, Novo Nordisk) 1,2 mg IV (15 $\mu \mathrm{g} / \mathrm{kg})$ was given. Within minutes, bleeding decreased drastically and formation of clot was observed. However, bleeding persisted around $200 \mathrm{ml} / \mathrm{h}$ and tranexamic acid 30mg/kg IV was given followed by a perfusion. Only 2 PRBC were given after the rFVIIa. Total losses were evaluated to 13,5L. The patient was released from the ICU 75 days later, after correction of several non-hemorragic complications. Discussion: The use of DDAVP did not decrease the bleeding as it has been reported in OI in literature(2). The only application of rFVIIa in hemorrhage in OI was successful with $40 \mu \mathrm{g} / \mathrm{kg}(3)$. The doses reported in literature vary from 20 to $200 \mu \mathrm{g} / \mathrm{kg}(4)$. We opted for a low dose regimen to diminish the risks of thromboembolic events(5). The dose of tranexamic acid used in our case is comparable to those cited in cardiac surgery context. Owing to OI, the risk of thrombosis in our patient probably was lowered because of the collagen anomaly. Of note, our patient never developed DIC although massive blood losses would have normally triggered maximal coagulation. Our observation shows that in massive intraabdominal hemorrhage secondary to surgery in the context of OI recombinant factor VIIa is effective at a lower dosage compared to that generally used in the off-label situations.

References: 1-Evensen, S.A. et al. Scand J Haematol 1984; 33: 177-179 2-Keegan MT, Whatcott BD, Harrison BA. Anesthesiology. 2002 Oct;97(4):1011-3 3-Kastrup M, von Heymann C, Hotz H, Konertz WF, Ziemer S, Kox WJ, Spies C. Ann Thorac Surg. 2002 Sep;74(3):910-2. 3-Mannuci, PM. Levi, M. N Engl J Med 2007; 356:2301-11. 4-Bauza, G. Transfusion 2007 :47; 749-750 


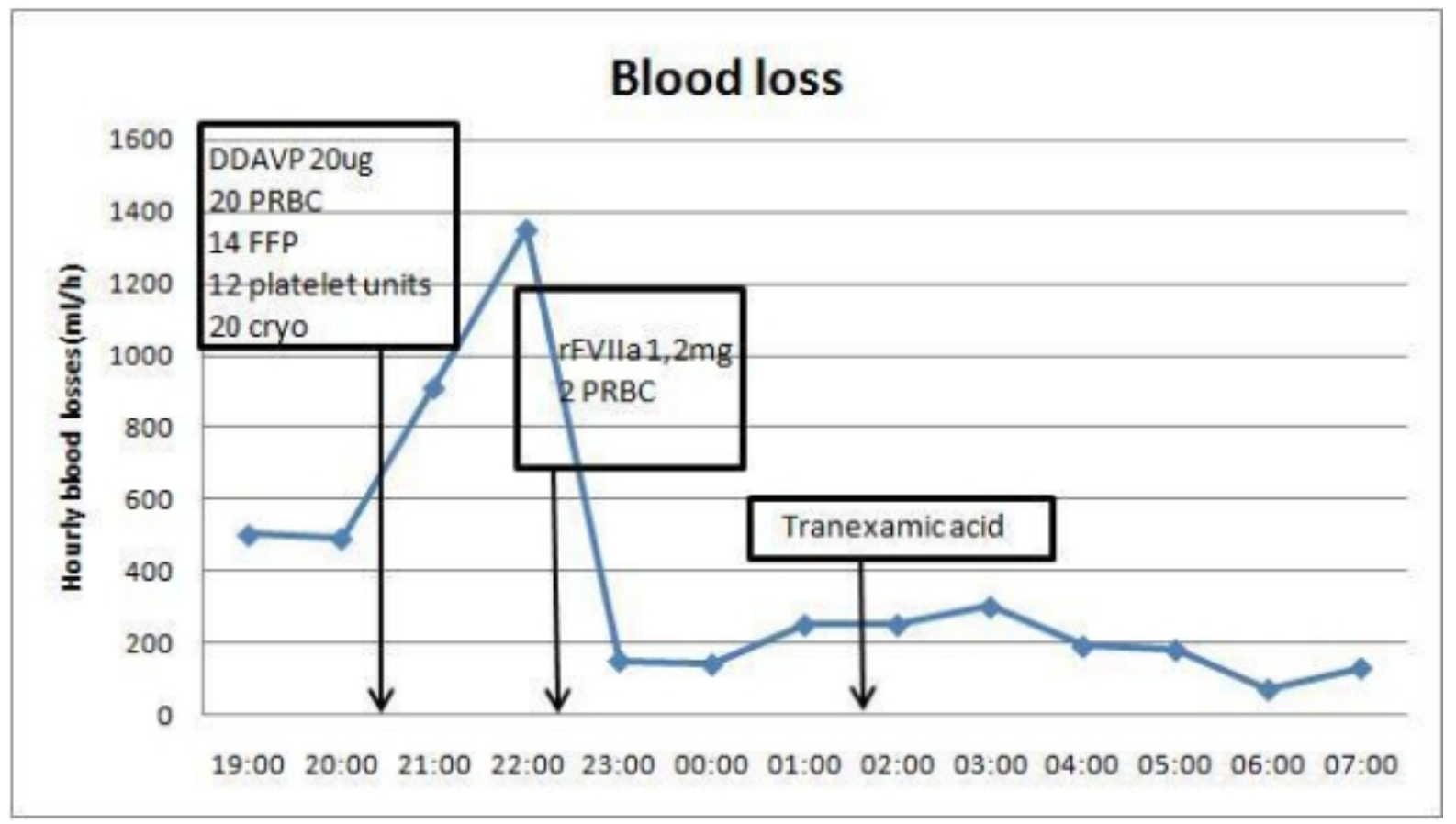

